\title{
Natural Convection Heat Transfer from Two Perpendicular Heated Surfaces Embedded In Porous Cavity
}

\author{
Dr. Amir Sultan Dawood \\ Asst. Prof. \\ College of Engineering- University of Mosul
}

\author{
Noor Moneer Basher \\ Asst. Lect. \\ Technical College- A/C Dept.
}

\begin{abstract}
The effect of modified Rayleigh number, positions and lengths of two perpendicular heated surfaces on natural convection heat transfer was studied numerically, where the two surfaces are embedded in square cavity filled with saturated porous medium. All walls of the cavity are kept at constant temperature. Indirect numerical method was used to solve the governing equations, which are: the nondimensional Darcy flow equation as well as the non-dimensional energy equation, which were solved numerically by finite difference method using Gauss-Seidel iteration coupled with (Successive Under Relaxation) technique. This study covered a wide range of modified Rayleigh number range (100-1000), nine positions and the ratios of length of vertical surface to the horizontal $(0.5,1,2)$. It was found that the positions of two heated surfaces have small effect on the heat transfer rate, but any increase in the length of two surfaces leads to an increase in average Nusselt number, but an increase in the length of vertical surface leads to more increase in the average Nusselt number.

KEY WORDS: Natural Convection, Heated Surfaces, Porous Media, Cavity

إنتقال الحرارة بالحمل الطبيعي من سطحين مسخنين متعامدين مغمورين في فجوة مسامية

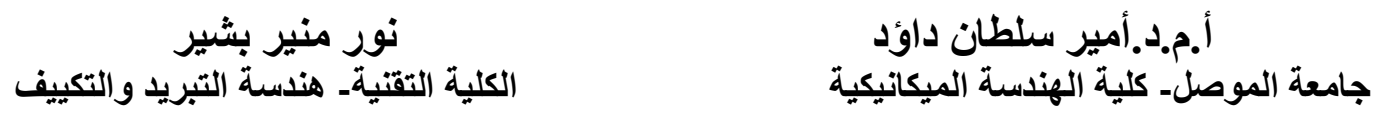

(الخلاصة

في هذا البحث تم إجراء دراسة عددية لبيان تأثير عدد رالي المطور ومواقع وطولي السطحين المسخنين

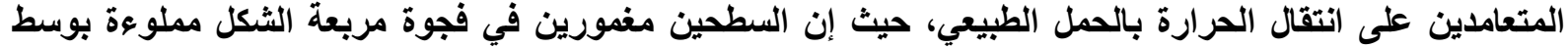

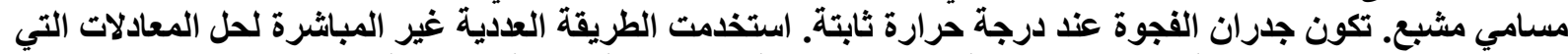

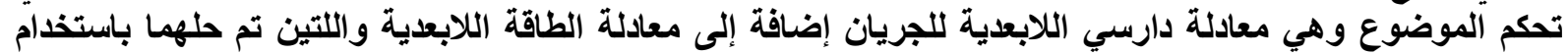

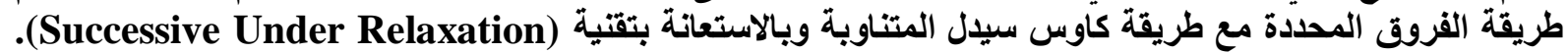

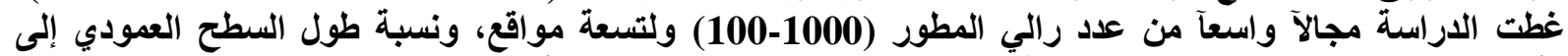

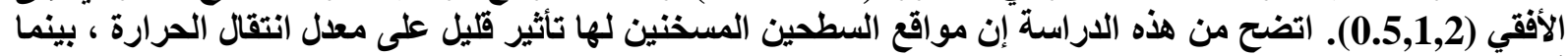

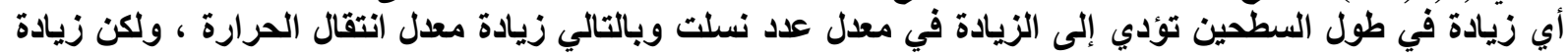
معدل عدد نسلت تكون اكبر بزيادة طول السطح العمودي.
\end{abstract}


Nomenclature

\begin{tabular}{|c|c|c|c|c|}
\hline $\mathrm{A}$ & Aspect ratio & & $\theta$ & Dimensionless temperature \\
\hline $\mathrm{Cp}$ & $\begin{array}{l}\text { Specific heat at constant } \\
\text { pressure }\end{array}$ & $\mathrm{J} / \mathrm{kg} . \mathrm{K}$ & $\psi$ & Stream function \\
\hline $\mathrm{g}$ & Gravitational acceleration & $\mathrm{m} / \mathrm{s} 2$ & & Subscript \\
\hline hc & Heat transfer coefficient & $\mathrm{W} / \mathrm{m} 2 . \mathrm{K}$ & Cold & $\mathrm{c}$ \\
\hline h1 & Location of vertical surface & $\mathrm{m}$ & effective & $\mathrm{e}$ \\
\hline $\mathrm{h} 2$ & Location of horizontal surface & $\mathrm{m}$ & $\mathrm{h}$ & \\
\hline ke & Effective thermal conductivity & $\mathrm{W} / \mathrm{m} . \mathrm{K}$ & o & \\
\hline $\mathrm{L}$ & Length of porous cavity & $\mathrm{m}$ & & Superscript \\
\hline L1 & Length of vertical surface & $\mathrm{m}$ & $\wedge$ & Dimensionless parameter \\
\hline L2 & $(\mathrm{L} 1=0.1 \mathrm{~L} 2)$ & $\mathrm{m}$ & & \\
\hline $\mathrm{Nu}$ & $\begin{array}{l}\text { Length of horizontal surface } \\
\text { (L2=0.1L1) }\end{array}$ & & & \\
\hline Qconv. & Nusselt number & $\mathrm{W}$ & & \\
\hline Qcond.. & Convection heat flow rate & $\mathrm{W}$ & & \\
\hline $\mathrm{Ra}^{*}$ & Conduction heat flow rate & & & \\
\hline $\mathrm{T}$ & Modified Rayleigh number & $\mathrm{K}$ & & \\
\hline $\mathrm{u}$ & Temperature & $\mathrm{m} / \mathrm{s}$ & & \\
\hline $\mathrm{v}$ & Fluid velocity in $\mathrm{x}$-direction & $\mathrm{m} / \mathrm{s}$ & & \\
\hline$\alpha$ & Fluid velocity in $y$-direction & $\mathrm{m} 2 / \mathrm{s}$ & & \\
\hline$\beta$ & Thermal diffusivity & $\mathrm{K}-1$ & & \\
\hline $\mathrm{v}$ & $\begin{array}{l}\text { Coefficient of thermal } \\
\text { expansion }\end{array}$ & $\mathrm{m} 2 / \mathrm{s}$ & & \\
\hline$\rho$ & Kinematic viscosity & $\mathrm{kg} / \mathrm{m} 3$ & & \\
\hline$\mu$ & density & $\mathrm{kg} / \mathrm{m} . \mathrm{s}$ & & \\
\hline
\end{tabular}

\section{1: Introduction}

Natural convection is one of the important modes of heat transfer. It occurs frequently as a result of density inversion caused by either the thermal expansion of a fluid, or the concentration gradients within a fluid system. This leads to generating the buoyancy force which driving the flow. Natural convection cooling is desirable because it doesn't require an energy source, such as a forced air fan and its maintenance free and safe. Natural convection can also happen in a porous medium saturated with a fluid. Generally, the porous medium means a material consisting of a solid matrix with an interconnected void. The solid matrix is supposed to be either rigid or it undergoes small deformation. The interconnectedness of the void allows the flow of one or more fluids through the material [1]. The porous media can be naturally formed (e.g. rocks, sponges) or fabricated (e.g. insulation, wicks) [2]. The analysis of heat transfer in porous media is required in a large number of applications. The applications are in the areas of chemical, mechanical and petroleum engineering. It is used in insulations, wicked heat pipes, storage of absorbed solar energy, nuclear reactors using gaseous coolants flowing through radioactive pellet $[3,4]$. Several studies have been carried out on free convection heat transfer for a vertical or a horizontal plate embedded in porous medium. Bejan \& Anderson [5] examined the buoyancy induced circulation occurring on both sides of a vertical impermeable partition separating two semi-infinite porous reservoirs maintained at different temperatures. But, Bejan [6] studied numerically the effect of internal 
flow obstruction on heat transfer through a two-dimensional porous layer heated from the sides. Three types of flow obstructions were considered. Nazar et al. [7] performed a similarity analysis of the steady free convection boundary layer over horizontal surface embedded in a fluid saturated porous medium with mixed thermal boundary conditions. Bansod \& Jadhav [8] analyzed the heat and mass transfer characteristics of natural convection a bout a horizontal surface embedded in a saturated porous medium. From the previous works, various studies had been published on the mechanism of convection heat transfer of vertical or horizontal plates separately, embedded in porous medium. However no work has been done on the vertical and horizontal plate together. So, the present work represented the natural convection heat transfer from two perpendicular heated surfaces with different dimensions and locations embedded in porous cavity at different modified Rayleigh numbers. The main object of this study is to investigate numerically the effect of the surfaces lengths as well as the locations on the natural convection heat transfer inside a porous cavity. The results will be represented graphically in terms of isotherms and streamlines.

\section{2: Mathematical Formulation}

Consider two normal isothermal surfaces at the same constant hot temperature $\left(\mathrm{T}_{\mathrm{h}}\right)$, embedded in a porous cavity of dimension $\mathrm{L} \times \mathrm{L}$, the walls of the cavity are kept at constant cold temperature $\left(T_{c}\right)$. The length of the hot surfaces is $L_{1}$ and $L_{2}$, respectively. The location of these surfaces from the right enclosure side and bottom is $h_{1}$ and $h_{2}$ respectively ,see figure (1).

The following assumptions are made in order to simplify the analysis of the problem:

1. The fluid is incompressible, the flow is steady and laminar.

2. A continuum saturated porous medium.

3. Viscous dissipation is neglected.

4. Heat generation is neglected.

5. Impermeable boundary surfaces.

6. Homogenous and isotropic properties of the fluid and solid matrix.

7. At any point in the porous medium, the solid matrix is in thermal equilibrium

with the fluid filling the pores.

8. Fluid properties are constant expect for the buoyancy term.

The equations that govern the heat transfer and fluid flow in a saturated porous medium are the continuity, momentum, and energy [3];

$$
\frac{\partial u}{\partial x}+\frac{\partial v}{\partial y}=0
$$

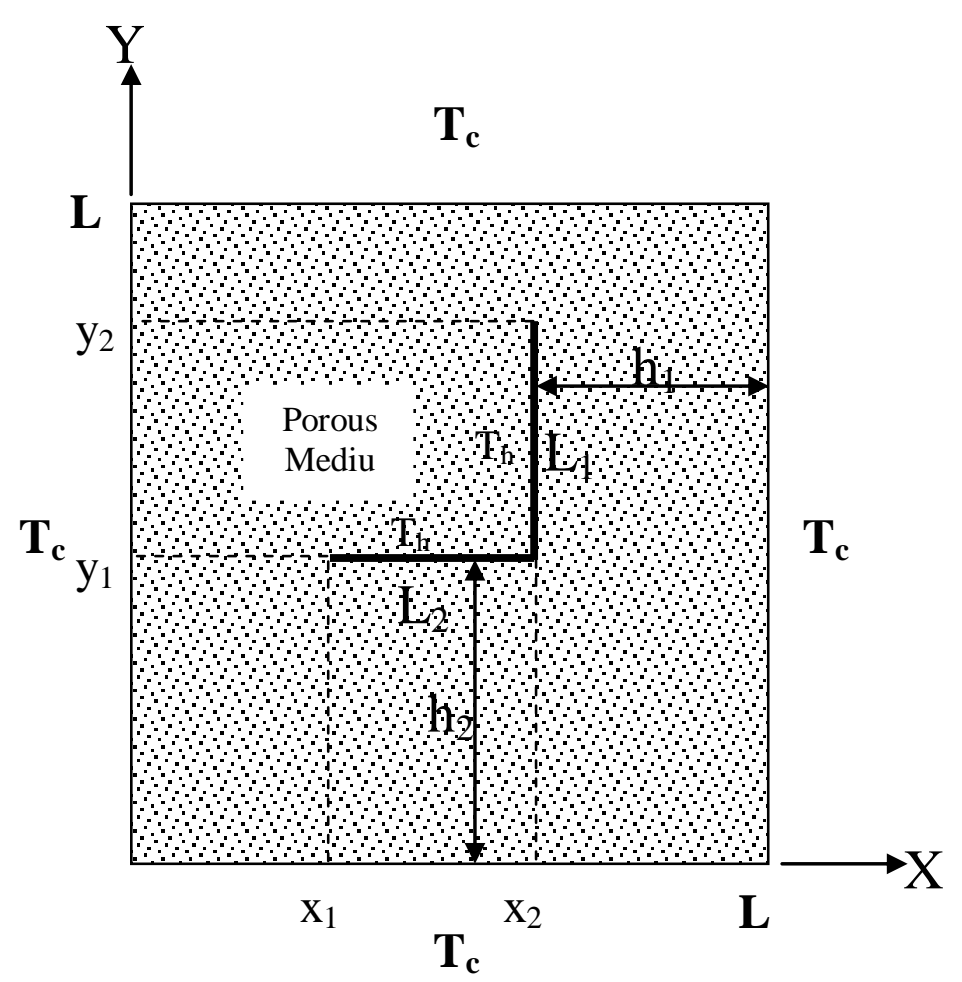

Figure (1): Physical problem 


$$
\begin{aligned}
& \frac{\partial u}{\partial y}-\frac{\partial v}{\partial x}=-\frac{\rho_{o} g K \beta}{\mu}\left[\frac{\partial T}{\partial x}\right] \\
& u \frac{\partial T}{\partial x}+v \frac{\partial T}{\partial y}=\alpha_{e}\left[\frac{\partial^{2} T}{\partial x^{2}}+\frac{\partial^{2} T}{\partial y^{2}}\right] \\
& \alpha_{e}=\frac{k_{e}}{\left(\rho \cdot C_{P}\right)_{f}}
\end{aligned}
$$

The boundary conditions are required to complete the formulation of the problem. The hot surface locations varied taking into consideration the horizontal distance from left vertical wall of the cavity to the center of the horizontal hot surface is $25 \%, 50 \%$ and $75 \%$ from the cavity width. Also the vertical distance from the bottom wall of the cavity to the horizontal hot surface is $25 \%, 50 \%$ and $75 \%$ of the cavity height, due to that nine positions are got as shown in figure (2).

\section{3: Dimensionless Formulation}

All the spatial dimensions are non-dimensionalized with respect to cavity dimensions $(\mathrm{L})$, then:

$$
\begin{gathered}
\mathrm{A}=\frac{\mathrm{L}}{\mathrm{L}}, \hat{x}=\frac{x}{L} \quad, \quad \hat{y}=\frac{y}{L}, \\
\hat{u}=\frac{u L}{\alpha_{e}} \quad, \quad \hat{v}=\frac{v L}{\alpha_{e}}, \quad \theta=\frac{T-T_{c}}{T_{h}-T_{c}} \\
\hat{L_{1}}=\frac{L_{1}}{L} \quad, \quad \hat{L_{2}}=\frac{L_{2}}{L}
\end{gathered}
$$

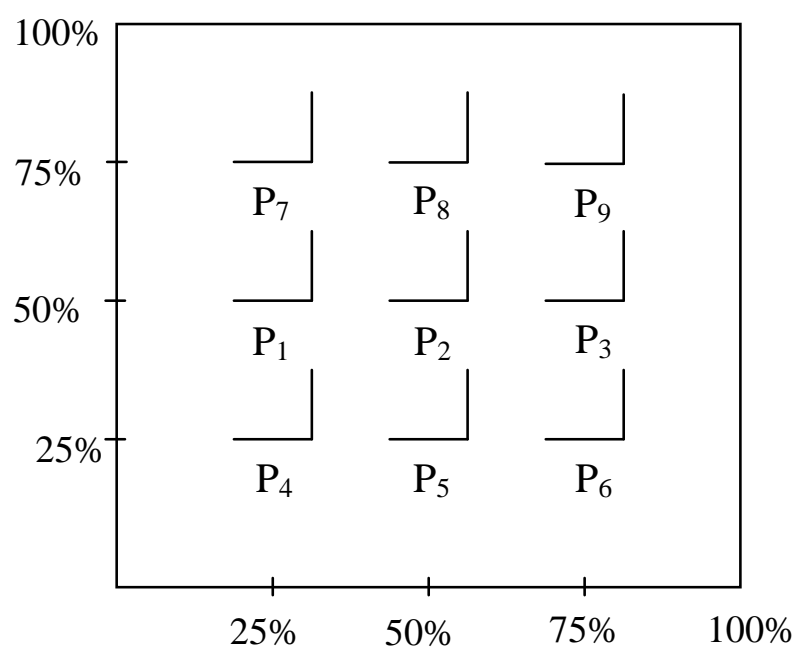

Figure (2): Different positions of the hot surfaces in a square cavity.

The stream function is defined in terms of the velocity components, which satisfies the continuity equation: [2]

$$
u=\frac{\partial \psi}{\partial y} \quad \text { and } \quad v=-\frac{\partial \psi}{\partial x}
$$

The stream function is non-dimensionalized as follow:

$$
\hat{\psi}=\frac{1}{\alpha_{e}} \psi
$$

The governing equations and the boundary conditions will take the following non-dimensional forms:

\section{3-1: Momentum Equation}

$$
\frac{\partial^{2} \hat{\psi}}{\partial \hat{x^{2}}}+\frac{\partial^{2} \hat{\psi}}{\partial \hat{y^{2}}}=-R a^{*}\left[\frac{\partial \theta}{\partial \hat{x}}\right]
$$


$R a^{*}=\frac{g \beta K L \Delta T}{v \alpha}$

\section{3-2: Energy Equation}

$$
\frac{\partial \hat{\psi}}{\partial \hat{y}} \frac{\partial \theta}{\partial \hat{x}}-\frac{\partial \hat{\psi}}{\partial \hat{x}} \frac{\partial \theta}{\partial \hat{y}}=\frac{\partial^{2} \theta}{\partial \hat{x^{2}}}+\frac{\partial^{2} \theta}{\partial \hat{y^{2}}}
$$

3-3: Boundary Conditions: The figure (3) shows the non-dimensional boundary condition for the square cavity, where:

$$
\begin{aligned}
& \text { at } \hat{x}=0,1 \quad \wedge \text { and } \quad \wedge \leq \hat{y} \leq 1 \\
& \theta=0 \quad, \quad \psi=0 \quad, \quad u=0 \\
& \text { for } \begin{array}{l}
\hat{y}=0,1 \\
\theta=0, \quad \hat{\psi}=0, \quad \hat{v}=0
\end{array}
\end{aligned}
$$

For the isothermal hot surfaces the dimensionless boundary conditions are:

$$
\begin{aligned}
& \text { at } \begin{array}{l}
\hat{y_{1}} \leq \hat{y} \leq \hat{y_{2}} \\
\hat{\theta}=1, \quad \text { and } \quad \hat{x}=0 \quad \hat{x}=\hat{x_{2}}
\end{array} \\
& \text { for } \quad \hat{x}_{1} \leq \hat{x} \leq \hat{x}_{2 \wedge} \text { and } \wedge \hat{y}=\hat{y}_{1} \\
& \theta=1 \quad, \quad \psi=0 \quad, \quad v=0
\end{aligned}
$$

Figure (3): Dimensionless boundary condition for the enclosure geometry.

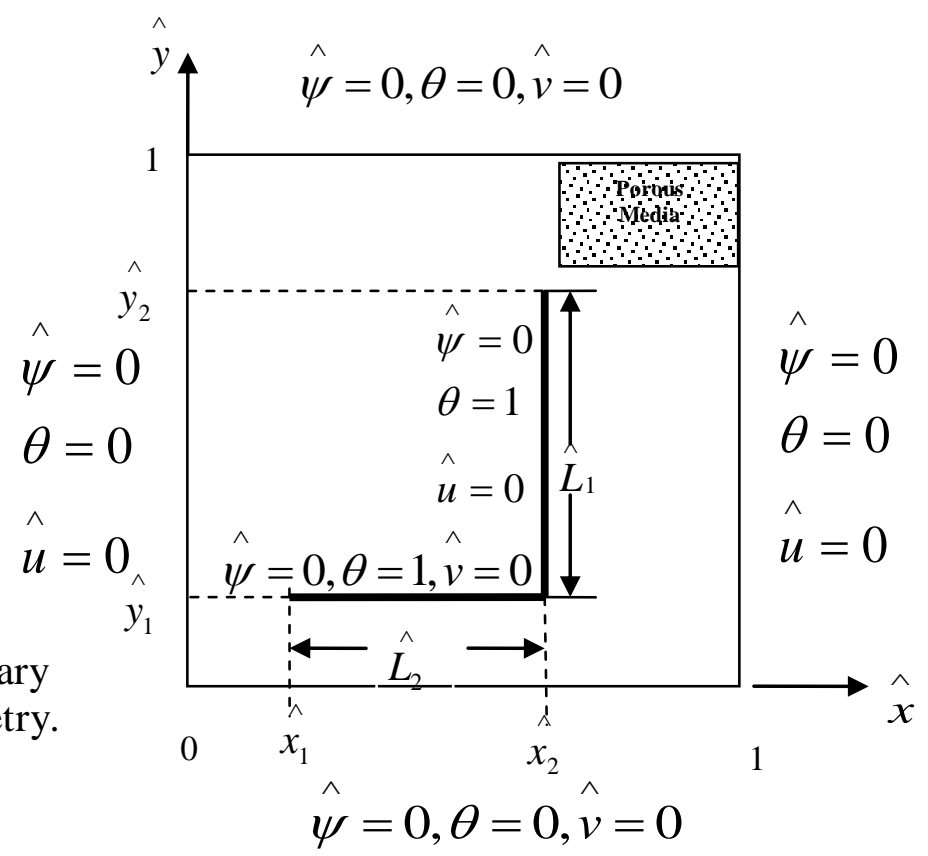

\section{4: Output Quantities}

Nusselt number, which is the ratio of the convective to conductive heat transfer, is an important output quantity [9]. 


$$
N u=\frac{Q_{\text {convection }}}{Q_{\text {conductio } 50}}
$$

Where (0) indicated zero Rayleigh number.

The convective heat transfer is calculated for the horizontal wall from:

$$
Q_{\text {conv }}=k_{e} \int_{0}^{L}\left(\frac{\partial T}{\partial y}\right)_{y=0, L} d x
$$

Where as the conductive heat transfer is calculated from:

$$
Q_{\text {cond }, 0}=k_{e} L \frac{\Delta T}{L}
$$

Thus, equation (15) become;

$$
N u=\frac{k_{e} \int_{0}^{L}\left(\frac{\partial T}{\partial y}\right)_{y=0, L} d x}{k_{e} L \frac{\Delta T}{L}}
$$

In dimensionless form, the equation becomes:

$$
N u=\int_{0}^{1}\left(\frac{\partial \theta}{\partial \hat{y}}\right)_{\hat{y}=0,1} d \hat{x}
$$

Similar forms exist for the vertical walls. The average Nusselt number

$(\overline{\mathrm{Nu}})$ is then calculated by averaging the local Nusselt numbers at the four walls [10], which the same Nusselt number being calculated for the hot surfaces using equations (19).

\section{5: Numerical Approach}

In the numerical approach, a fairly complete mathematical description of heat transfer phenomena, as well as it is capable of handling large systems of equations, nonlinearities, and complicated geometries that are often impossible to solve analytically, therefore the differential equations are solved numerically. The base of finite difference technique is to approximate all the partial derivatives in the equation using Taylor series expansion. For simplicity, we assume that both $\Delta x$ and $\Delta y$ are constant. Using the centered, forward, and backward differences approximation. After substituting the finite-difference in the non-dimensional governing equations, the following expressions are obtained:

\section{5-1: Momentum Equation}

$$
\frac{1}{(\Delta \hat{x})^{2}}\left(\hat{\psi}_{i-1, j}-2 \hat{\psi}_{i, j}+\hat{\psi}_{i+1, j}\right)+\frac{1}{(\Delta \hat{y})^{2}}\left(\hat{\psi}_{i, j-1}-2 \hat{\psi}_{i, j}+\hat{\psi}_{i, j+1}\right)=-R a^{*}\left[\frac{1}{2 \Delta \hat{x}}\left(\theta_{i+1, j}-\theta_{i-1, j}\right)\right]
$$

\section{5-2: Energy Equation}

$$
\frac{1}{2 \Delta \hat{y}}\left(\hat{\psi}_{i, j+1}-\hat{\psi}_{i, j-1}\right) \cdot \frac{1}{2 \Delta \hat{x}}\left(\theta_{i+1, j}-\theta_{i-1, j}\right)-\frac{1}{2 \Delta \hat{x}}\left(\hat{\psi}_{i+1, j}-\psi_{i-1, j}\right) \cdot \frac{1}{2 \Delta \hat{y}}\left(\theta_{i, j+1}-\theta_{i, j-1}\right)=
$$




$$
\frac{1}{(\Delta \hat{x})^{2}}\left(\theta_{i-1, j}-2 \theta_{i, j}+\theta_{i+1, j}\right)+\frac{1}{(\Delta \hat{y})^{2}}\left(\theta_{i, j-1}-2 \theta_{i, j}+\theta_{i, j+1}\right)
$$

Then the above equations are re-arranged in a form that enables them to be handle by a computer, where the computer program is written in the (Fortran 90) language by using program (Fortran Power Station). This program was used on different numbers of grid points, modified Rayleigh and aspect ratio. The grid independence of the results must be tested. The modified Rayleigh number in this test is set to be 1000 , while the grid size varies from $20 * 20$ to $90 * 90$ and at aspect ratio of 1.0 , as shown in figure (4).

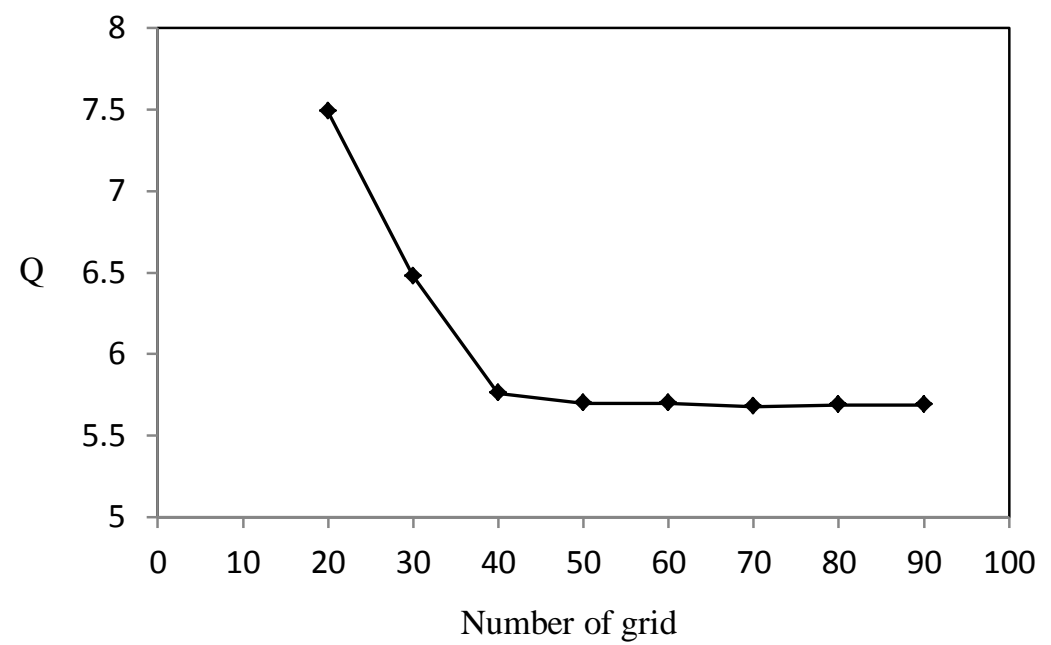

Figure (4): Variation of heat flow rate vs. number of grid at $\mathrm{Ra}^{*}=1000$.

It was found that the change in the heat flow rate for grid size of $40 * 40$ and $50 * 50$ is less than $0.1 \%$. Therefore, the number of points (grid) that is adopted in the present study is $40 * 40$. The number of points was selected as a compromise between accuracy and speed computation. Using the Gauss Siedel iterative method. The convergence rate of the GaussSeidel iterative procedure can often be improved by applying "acceleration" procedures (reduce computation time). The simplest of these is known as Successive (Over \& Under Relaxation), used Successive Under Relaxation (SUR). Those methods are used to find the actual value of temperature and stream-function with an accuracy of $\sum\left|T N_{i, j}-T_{i, j}\right| \leq 10^{-4}$.

\section{6: Validation.}

The comparison problem is simply convective heat transfer in a two-dimensional porous cavity, subject to different temperatures on its vertical sides, while the horizontal sides are perfectly insulated. Table shown below, show comparisons at two different values of modified Rayleigh number 50 and 100, and at aspect ratio of 1.0. The results agree with the present results with a maximum difference of $2 \%$ at $R a^{*}=100$, these differences are attributed to the finite difference approximation. 


\begin{tabular}{|l|c|c|c|c|c|c|}
\hline \multirow{2}{*}{ A } & & \multicolumn{5}{|c|}{ Nu } \\
\cline { 3 - 7 } & Ra* $^{*}$ & $\begin{array}{c}\text { Chan } \\
\text { et al. [11] }\end{array}$ & $\begin{array}{l}\text { Dawood\& } \\
\text { Burns [12] }\end{array}$ & $\begin{array}{c}\text { Bejan\& } \\
\text { Tien [13] }\end{array}$ & $\begin{array}{c}\text { Burns } \\
\text { et al [14] }\end{array}$ & $\begin{array}{c}\text { Present } \\
\text { Work }\end{array}$ \\
\hline \multirow{3}{*}{1.0} & 50 & 2.100 & 2.220 & --- & 2.200 & 2.11 \\
\cline { 2 - 7 } & 100 & 3.560 & 3.472 & 3.600 & 3.600 & 3.54 \\
\hline
\end{tabular}

\section{7: Results.}

The main purpose of this study is to investigate the effect of the two perpendicular isothermal hot surfaces lengths, as well as the positions on the natural convection heat transfer inside a porous cavity. The investigation parameters are: modified Rayleigh numbers ( $100 \leq R a^{*} \leq 1000$ ), nine positions of hot surfaces, and the lengths of hot surfaces either equal or different.

\section{7-1: The hot surfaces with the same lengths}

7-1-1: The hot surfaces in the mid height of cavity $\left(\mathbf{P}_{\mathbf{1}}, \mathbf{P}_{\mathbf{2}}, \mathbf{P}_{\mathbf{3}}\right)$ : The positions of hot surfaces in the mid height of cavity which is represented by $\left(\mathrm{P}_{1}, \mathrm{P}_{2}\right.$, and $\left.\mathrm{P}_{3}\right)$ in figure (2). A horizontal distance from left vertical wall of the cavity to the center line of the horizontal hot surface is $25 \%, 50 \%$ and $75 \%$ from the cavity width, respectively. The vertical distance between the bottom wall of the cavity and the horizontal hot surface is $50 \%$ of the cavity height. Figure (5) illustrate the effect of hot surfaces for these positions on the streamlines and isotherms, at different modified Rayleigh numbers. Two counter-rotating cells, the left cell rotates counter-clockwise, but the other cell rotates clockwise and each cell rotates around a point of maximum stream functions $\left(\psi_{\max }\right)$, cells formed due to presences of hot surfaces. The warm fluid is rising slightly in the vicinity from the hot surfaces, turning at the top of the cavity, moving adjacent the cold wall, turning, and descending along the two vertical walls of the cavity. The result for this motion of flow causes the circulating motion for the cells. The maximum value of stream function can be viewed as a measure of the intensity of natural convection in the cavity.

The effect of changing modified Rayleigh number on the isotherms at changing the positions of hot surfaces shown in the same figure. It is clear that the isotherms lines start to occupies all regions above it, and happen when increased the value of $\mathrm{Ra}^{*}$. This is referred to increase the rate of heat transfer by natural convection.

7-1-2: The hot surfaces in the lower part of cavity $\left(\mathbf{P}_{\mathbf{4}}, \mathbf{P}_{\mathbf{5}}, \mathbf{P}_{\mathbf{6}}\right)$ : Figures $(6-\mathrm{a} \&$ 6-b) give an idea about the streamlines and isotherms for $\mathrm{Ra}^{*}=1000$. Streamlines and isotherms have similar behavior that illustrated in aforementioned case. Generally, in these positions, the region above hot surfaces is larger which allows more flow circulation. In addition to that, streamlines and isotherms start filling the entire cavity with increase in value of $\psi_{\max }$ as compared with case one. On the other hand, it reveals that conduction effect becomes insignificant below the hot surfaces. This led to large amount of heat transfer by natural convection from the hot surfaces to the walls of cavity 

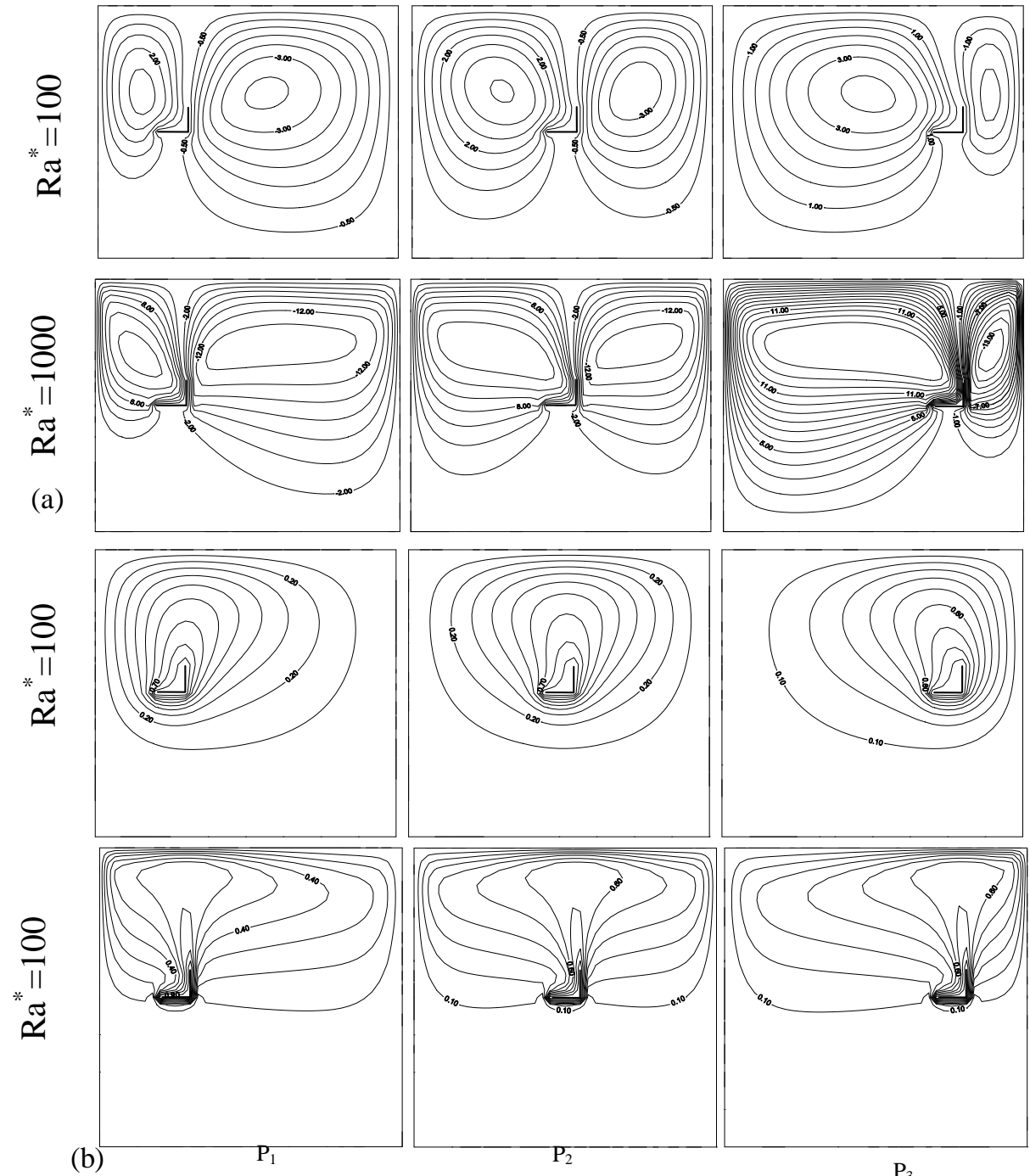

Figure (5): Effect of the hot surfaces on (a) streamlines; (b) isotherms at different $\mathrm{Ra}^{*}$.

(a)
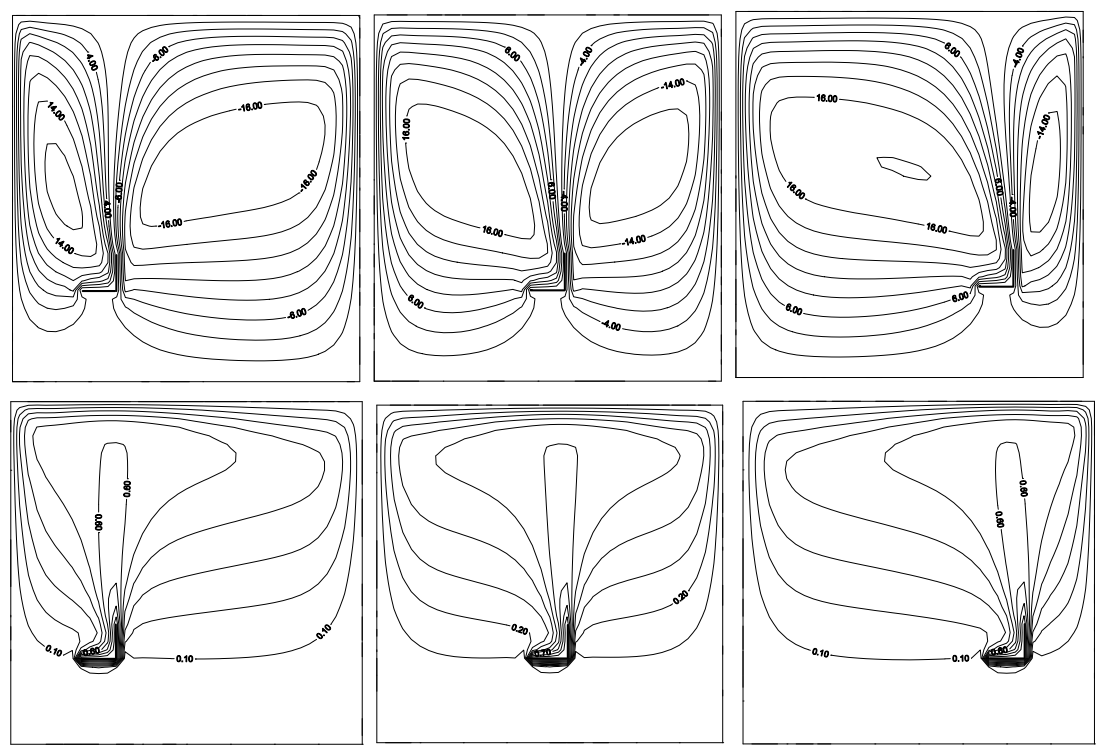

Figure (6): Effect of the hot surfaces on (a) streamlines; (b) isotherms for $\mathrm{L}_{1}=\mathrm{L}_{2}$. 
7-1-3: The hot surfaces in the upper part of cavity $\left(\mathbf{P}_{7}, \mathbf{P}_{8}, \mathbf{P}_{9}\right)$ : Also, the same mode of previous cases take streamlines and isotherms, but the upward movement of hot surfaces leads to flow suppression due to the small area above it, causing decrease from the rate of heat transfer. This movement reducing the value of maximum stream functions $\psi_{\text {max }}$ as compared to those cases, consequently decreases of heat transfer by natural convection, as shown in Fig. (7).

(a)
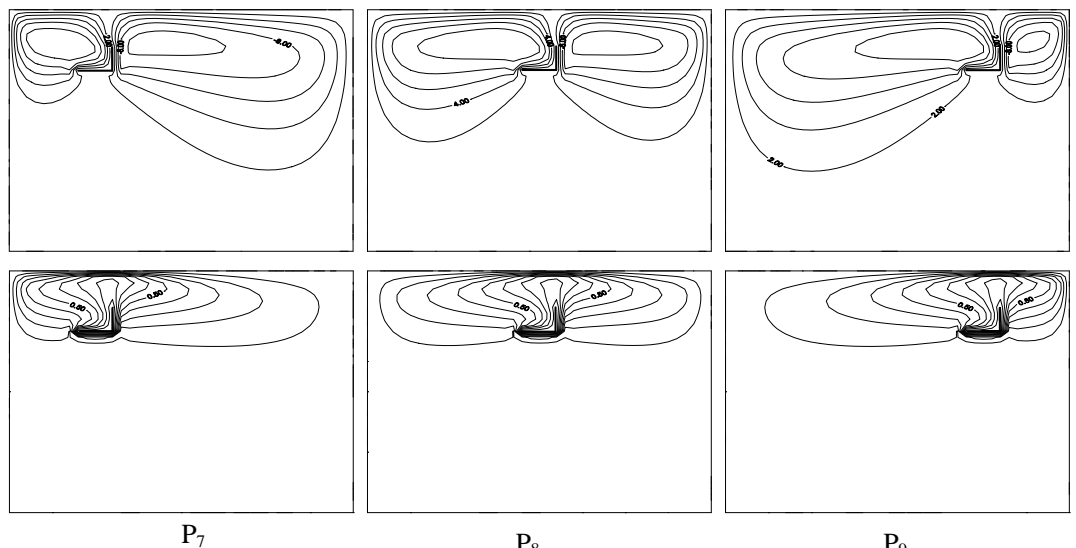

Figure (7): Effect of the hot surfaces on (a) streamlines; (b) isotherms for $\mathrm{Ra}^{*}=1000, \mathrm{~L}_{1}=\mathrm{L}_{2}$

7-2: The hot surfaces with different lengths: Figures $(8, a-b)$ illustrate the effect of different lengths of hot surfaces at $\mathrm{Ra} * 1000$. When the length of vertical or horizontal hot surfaces is increased, the rotation of the fluid is strong, due to the further amount of fluid exposes to heat and this proportionately produces large buoyancy force inducing convection. The extra heating effect is promoted as the length of vertical or horizontal hot surfaces are increased.

(a)
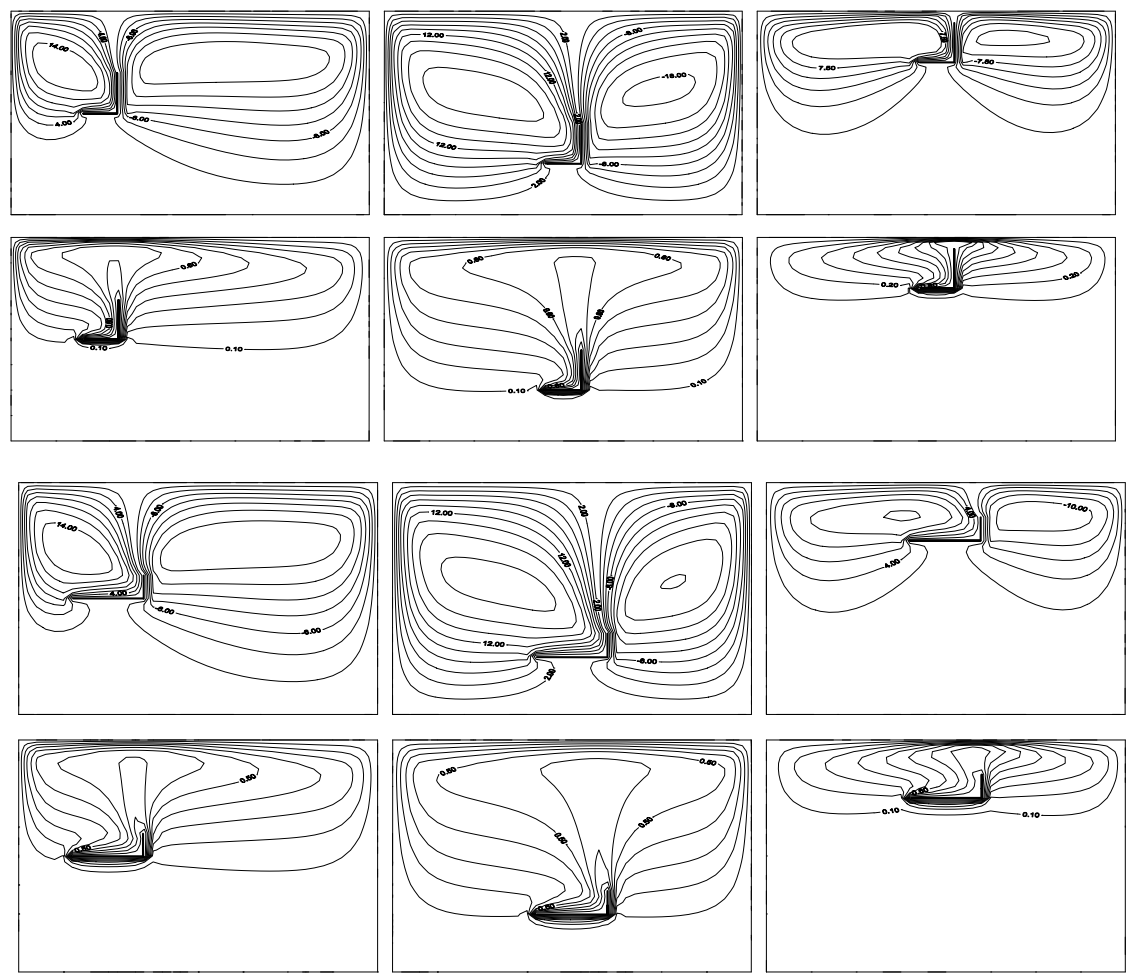

$\mathrm{P}_{1}$

$\mathrm{P}_{5}$

$\mathrm{P}_{8}$

Figure (8): isograms at $\mathrm{Ra}^{*}=1000$ : (a) $\mathrm{L}_{1}=2 \mathrm{~L}_{2}$; (b) $\mathrm{L}_{1}=0.5 \mathrm{~L}_{2}$ for different 
7-3: Heat transfer results: This section presents the effects of modified Rayleigh number, length and the positions of isothermal hot surfaces on the relative increase in the rate of heat transfer (average Nusselt number) graphically. Average Nusselt number is plotted as a function of hot surface positions at different values of $R a^{*}$ explained in figure (9). Values of average Nusselt number increased with the increase of $R a^{*}$ in the same position of hot surfaces. It can be observed that placing hot surfaces $\left(\mathrm{P}_{8}\right)$ reduces from the value of average Nusselt number. This is because the hot surfaces in this position block the flow above it. On the other hand, flow inhibition as a result diminishes from the rate of heat transfer in $\left(\mathrm{P}_{8}\right)$. For those reasons $\left(\mathrm{P}_{8}\right)$ is regarded as the best position to minimize the heat transfer by natural convection. This position might be used for the best insulation in such case.

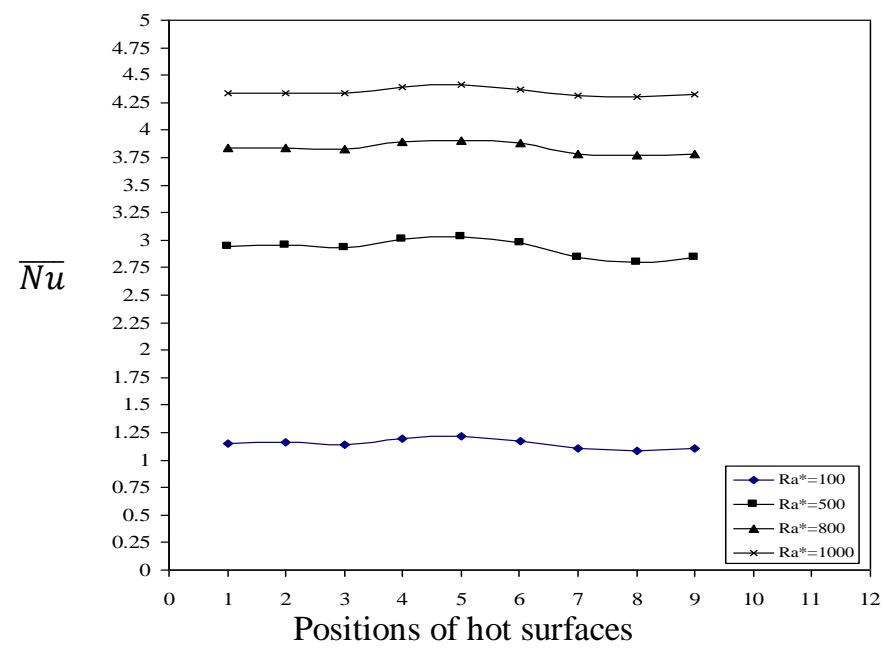

Figure (9): Variation of average Nusselt number vs. positions o hot surfaces for different modified Rayleigh number.

In figure (10) it can be observed that increasing $R a^{*}$ enhances $\overline{N u}$. This figure shows that $\overline{N u}$ is independent of the positions of hot surfaces, because it is revealed that there is a slight increase of average Nusselt number with positions of hot surfaces. As the results, positions of hot surfaces have small effect on the rate of heat transfer.

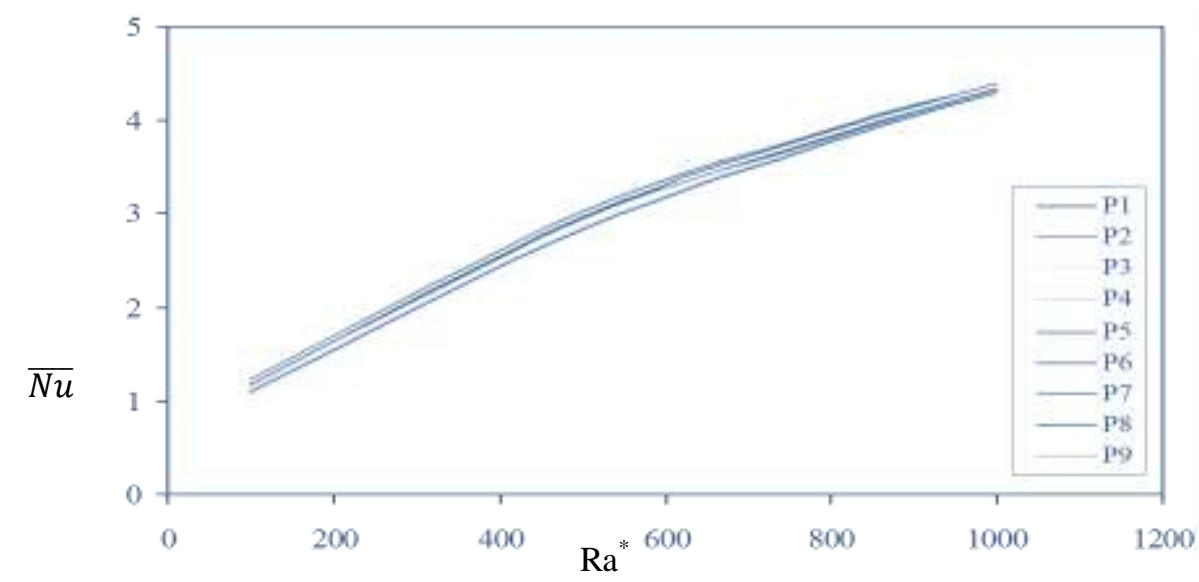

Figure (10): Variation of average Nusselt number with modified Rayleigh number for different positions of hot surfaces. 
Fig. (11) shows $\overline{N u}$ for different values of $R a^{*}$ and different lengths of vertical and horizontal hot surface at each position. It was found that $\overline{N u}$ increases with the increase in $R a^{*}$ and length of vertical hot surface in each position. In general, the length of hot surfaces plays a vital role in the overall heat transfer rate, specially the length of vertical hot surface regardless of its position.
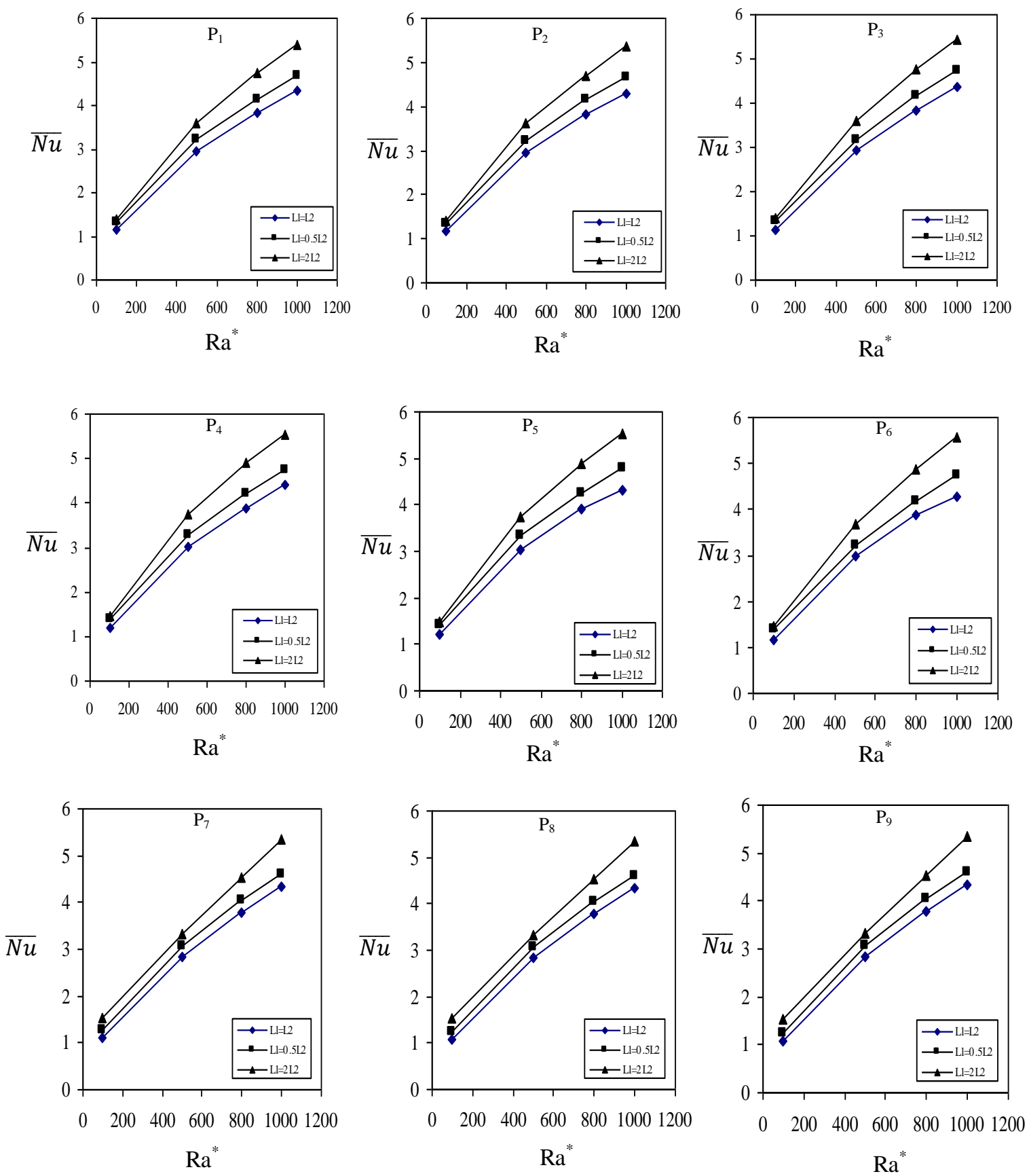

Figure (11): Variation of average Nusselt number vs. modified Rayleigh number at different lengths of hot surfaces. 


\section{8: Conclusions.}

The main conclusions of the present study are:

1. Average Nusselt number increases with the increase of the lengths of vertical and horizontal hot surfaces.

2. Positions of hot surfaces have small effect on the rate of heat transfer.

3. The maximum heat transfer occurs by increasing length of vertical hot surface.

\section{9: References.}

1. Neiled, D. A. and Bejan, A., "Convection in Porous Media," Springer, New York, Third Edition, (2006).

2. Vafai, K., "Handbook of Porous Media," Second Edition, Taylor \& Frances group, Bokarton, (2005).

3. Cheng, P., "Heat Transfer in Geothermal Systems," Adv. Heat

Transfer Vol. 14, pp.1-105, (1978).

4. Kaviany, M., "Principles of Heat Transfer in Porous Media,"

Chap. 1-2, Second Edition, (1998).

5. Bejan, A. and Anderson, R., "Heat Transfer Across a Vertical Impermeable Partition Embedded in Porous Medium," Int. J. Heat Mass Transfer, Vol. 24, No.7, pp.1237-1245, (1981).

6. Bejan, A., "Natural Convection Heat Transfer in a Porous Layer with Internal Flow Obstructions," Int. J. Heat Mass Transfer, Vol. 26, pp.815-822, (1983).

7. Nazar, R., Arifin, N. M. and Pop. I, "Free Convection Boundary Layer Flow Over Vertical and Horizontal Flat Plates Embedded in a Porous Medium Under Mixed Thermal Boundary Conditions," Int. Comm. Heat Mass Transfer, Vol. 33, pp. 87-93, (2006).

8. Bansod, V. J. and Jadhav, R. K., "An Integral Treatment for Combined Heat and Mass Transfer by Natural Convection Along a Horizontal Surface in a Porous Medium," Int. J. Heat Mass Transfer, Vol. 52, pp. 2802-2806, (2009).

9. Bejan, A., "Convection Heat Transfer," Wiley-Interscience

Publication, John Wiley \& Sons, Inc., (1995).

10. Kandaswamy, P., Lee, J., and Abdul Hakeem, A. K., "Natural Convection in a Square Cavity in The Presence of Heated Plate," Nonlinear Analysis: Modeling and Control, Vol. 12, No. 2, pp. 203-212, (2007).

11. Chan, B. K. C., Ivey, C. M., and Barry, J. M., "Natural Convection In Enclosed Porous Media With Rectangular Boundaries," J. Heat Transfer, Vol. 2, pp. 21-27, (1970).

12. Dawood, A. S. and Burns, P. J., "Steady Three-Dimensional Convective Heat Transfer in a Porous Box Via Multigrid," J. Numerical Heat Transfer, Vol. 22, pp. 167-198, (1992).

13. Bejan, A. and Tien, C. L., "Natural Convection in Horizontal Porous Media Subjected to an End-to-End Temperature Deference," J. Heat Transfer, Vol. 100, pp. 191-198, (1978).

14. Burns, P. J., Chow, L. C. and Tien, C. L., "Convection in Vertical Slot Filled With Porous Insulation,” Int. J. Heat Mass Transfer, Vol. 20, pp. 919-926, (1977). 